\title{
Comprehensive Benefit Evaluation based on Interaction of Micro Grid and Distribution Network
}

\author{
Weiping Zhu ${ }^{1}$, Xiaodong Yuan ${ }^{2}$, Yunpeng $\mathrm{Li}^{3}$ \\ 1. Jiangsu Electric Power Company, Nanjing, China,zwp_xjtu@163.com; \\ 2. Jiangsu Electric Power Company, Nanjing, China, lannyyuan@hotmail.com; \\ 3. Nantong Power Supply Company, Nantong, China,leeyunpeng@sina.com.
}

\begin{abstract}
Interaction between micro grid and distribution network plays an important role in improving absorptive capacity of new energy, increasing utilization ratio of distribution network assets and promoting the safe, economy, environmental operation of power system. This paper mainly researched comprehensive benefit of different interaction models of micro grid and distribution network, firstly, classic interaction ways were studied, index system of comprehensive benefit was determined, then index weight was determined by analytic hierarchy process(AHP), at last, comprehensive benefit of different interaction models were analyzed according to fuzzy comprehensive evaluation, which could offer decision-making for interaction of micro grid and distribution network.
\end{abstract}

Key words: Micro grid, distribution network, interaction, comprehensive benefit

\section{Introduction}

Micro grid is a kind of independent and decentralized network, which is made up of solar power generation, wind power generation, hydropower, biomass generation, gas power generation, fuel cell and battery casually, including metering devices and controlling devices. It is independent of public grid or connected to the public grid intermittently. Distribution Network ${ }^{[1-3]}$ is that whose voltage is $10 \mathrm{kV} \sim 110 \mathrm{kV}$, which has treelike, annular and reticulate connection modes, it is an important part in the power system facing the users. With the micro grid technology getting more and more mature, when the micro grid is connected to the distribution network, we hope it can improve electricity characteristic of distribution network, and reduce operating losses to realize efficient utilization of energy. As information technology develops, interaction between micro grid and distribution network becomes more and more close, not only exchange of power, but also exchange of information becomes more and more close ${ }^{[4-6]}$.

Comprehensive benefit of different interaction models of micro grid and distribution network can provide scientific basis for investment decision of micro grid, which is essential to the development of micro technologies and development of distribution network building.

Domestic and foreign scholars have made some achievements about research on interaction between micro grid and distribution network, literature[7] established a gray comprehensive evaluation model of micro-grid and experimental verification was carried out; literature[8] evaluated impact micro grid had on the reliability of the distribution network by analyzing the improvement of reliability with the micro grid connected to distribution network; literature[9] analyzed the economic benefits smart micro grid technologies provided for the power system and the participation subject, it also built the evaluation model of principal component analysis with compulsory score. However, it is not perfect enough lacking of sound evaluation scheme about comprehensive benefit based on interaction of micro grid and distribution network. 
This paper took comprehensive benefit based on interaction of micro grid and distribution network as research object, firstly classic interaction ways were studied, index system of comprehensive benefit was determined, including economic benefit, social benefit and technical benefit. Then index weight was determined by analytic hierarchy process, at last, comprehensive benefit of different interaction models were analyzed according to fuzzy comprehensive evaluation.

\section{Different Interaction Models}

As for the power network, interaction between micro grid and distribution network ${ }^{[10-12]}$ mainly reflects in three aspects: economic load distribution in normal situation; modulation of the load at the peak load when the load is too high; support of the main grid in emergency. Interaction between micro grid and distribution network is aimed at improving the ability of the grid to taking in new energy power, increasing utilization ratio of distribution network assets and bettering the safe, economy, environmental operation of power system. Different interaction models of micro grid and distribution network lie in the goals of coordinated cooperation.

Different interaction models of micro grid and distribution network are: firstly, interaction model based on economic goal; secondly, interaction model based on social goal; thirdly, interaction model based on technical goal.

The economic benefit of micro grid is the key point of attracting users and being promoted in the power system, economical operation of micro grid is an important method of realizing the economic benefit ${ }^{[13]}$, interaction model based on economic goal emphatically considers the benefit of peak load shifting, the benefit of reducing fuel cost, the benefit of reducing investment cost of the grid, selling benefit of the grid, the benefit of delaying electric grid invests and the benefit of reducing spinning reserve in the grid.

Spreading use of micro grid plays a positive role in user experience, environmental improvement, social progress, interaction model based on social goal emphatically considers environmental benefit, promoting energy conversion efficiency and reducing electric cost expenditure of users.

Interaction between micro grid and distribution network can reduce the loss of operation of the grid, increase utilization ratio of distribution network assets and promote the safe, efficient, environmental operation of power system ${ }^{[14-16]}$, interaction model based on technical goal emphatically considers the benefit of power supply reliability, the benefit of loss reduction and power quality.

\section{Analytic Hierarchy Process}

The steps of weight determination by analytic hierarchy process ${ }^{[17-18]}$ are as below:

(1) Construct judgment matrix. A represents the goal, $\mathrm{u}_{\mathrm{i}}, \mathrm{u}_{\mathrm{j}}(\mathrm{i}, \mathrm{j}=1,2 \ldots, \mathrm{n})$ are factors. $u_{i j}$ represents the importance $u_{i}$ means to $u_{j}$. Judgment matrix is made up of $u_{i j}$.

(2) Calculate importance ranking. Calculate the eigenvector $\omega$ corresponding to maximum characteristic root $\lambda_{\max }$ according to the judgment matrix.

$P \omega=\lambda_{\max } \omega$

$\mathrm{P}$ is the judgment matrix, after normalization the eigenvector $\omega$ is importance ranking of evaluation factors, which is also the weight distribution.

(3) Consistency check. Calculate the maximum characteristic root corresponding to every eigenvector. Then consistency check is carried out by using consistency index, random index and coincidence coefficient. The formula is as below:

$C R=C I / R I$

$\mathrm{CR}$ is coincidence coefficient of judgment matrix; $\mathrm{CI}$ is the general coincidence 
coefficient. It is shown as below:

$$
C I=\left(\lambda_{\max }-n\right) /(\mathrm{n}-1)
$$

$\mathrm{RI}$ is the average random index of judgment matrix, the value of RI from first order to ninth order is shown in table1.

Table 1. The value of RI of Random Index

\begin{tabular}{llllll}
\hline $\mathrm{n}$ & 1 & 2 & 3 & 4 & 5 \\
\hline $\mathrm{RI}$ & 0 & 0 & 0.50 & 0.90 & 1.12 \\
$\mathrm{n}$ & 6 & 7 & 8 & 9 & \\
$\mathrm{RI}$ & 1.24 & 1.32 & 1.41 & 1.45 & \\
\hline
\end{tabular}

When CR is smaller than 0.1 or $\lambda_{\max }$ is equal to $\mathrm{n}$, if $\mathrm{CI}$ is equal to zero, satisfaction of $\mathrm{P}$ is considered perfect, or the factors of $\mathrm{P}$ need to be adjusted.

\section{Fuzzy Comprehensive Evaluation}

Mathematical models of fuzzy comprehensive evaluation ${ }^{[19-20]}$ can be divided into first grade model and multilevel model, in this paper multilevel model is adopted. The steps of mathematical models adopting multilevel model is shown as below:

(1) Clarify factor levels. Divide the organization of factors $U$ into $m$ subset.

$$
U=\left\{u_{1}, u_{2}, \cdots u_{i}, \cdots u_{m}\right\} \quad(\mathrm{i}=1,2, \cdots, m)
$$

$u_{i}$ is the $\mathrm{i}$-th factor in the first floor, which is determined by the $\mathrm{n}$-th factor in the second floor:

$$
U_{i}=\left\{u_{i 1}, u_{i 2}, \cdots, u_{i j}, \cdots u_{i n}\right\}(j=1,2, \cdots, n)
$$

(2) Set up weight set. Provide corresponding weight value for every factor according to their importance value.

(3) Set up alternative set V. Alternative set is a collection of evaluation results valuator may give to evaluation object. Alternative set can be represented as below:

$$
V=\left\{v_{1}, v_{2}, \cdots, v_{p}\right\}
$$

In this paper five levels of "good, well, medium, poor, bad" are adopted.

(4) First grade model. Concerning that the factors in the first floor are determined by factors in the second floor, so single factor evaluation of every factor in the first floor should be results of multi factors evaluation in the second floor. Make single factor evaluation matrix in the second floor as $\mathrm{R}_{\mathrm{i}}$ :

$$
R_{i}=\left[\begin{array}{cccc}
r_{i 11} & r_{i 12} & \ldots & r_{i 1 p} \\
r_{i 21} & r_{i 22} & \ldots & r_{i 2 p} \\
\vdots & \vdots & & \vdots \\
r_{i n 1} & r_{i n 2} & \ldots & r_{i n p}
\end{array}\right]
$$

Row number of $R_{i}$ is determined by the number of evaluation factors in $r_{i j}$. First grade fuzzy comprehensive evaluation set $\mathrm{B}_{\mathrm{i}}$ can be got concerning weights: $B_{i}=A_{i} \cdot \mathrm{R}_{i}$

$$
\begin{aligned}
& =\left[a_{i 1}, a_{i 2}, \cdots, a_{i n}\right] \cdot\left[\begin{array}{cccc}
r_{i 11} & r_{i 12} & \ldots & r_{i 1 p} \\
r_{i 21} & r_{i 22} & \ldots & r_{i 2 p} \\
\vdots & \vdots & & \vdots \\
r_{i n 1} & r_{i n 2} & \cdots & r_{i n p}
\end{array}\right] \\
& =\left[b_{i 1}, b_{i 2}, \cdots, b_{i p}\right] \\
& \text { In formula (4), “. ” is fuzzy operator. }
\end{aligned}
$$


(5) Second grade fuzzy comprehensive evaluation. No matter how many floors there are, comprehensive evaluation results of the highest floor should be acquired. First-floor fuzzy comprehensive evaluation is just the results of the lowest floor. To calculate evaluation results of higher floor, second-floor fuzzy comprehensive evaluation should be carried out.

Composition of two floors can be sown in Picture 1:

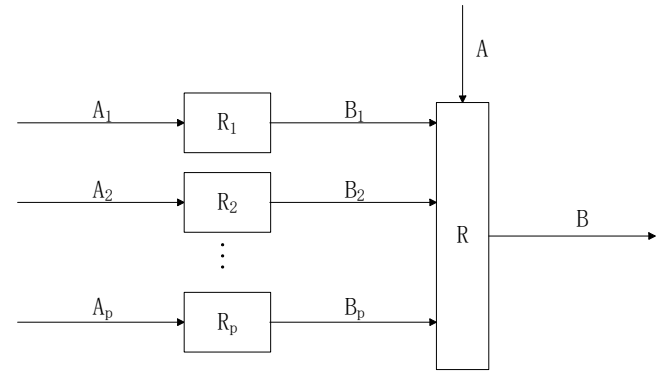

\section{Picture 1. Schematic Diagram of Second Grade Fuzzy Comprehensive Evaluation}

Once given transformation matrix of the lowest floor and weight values matrix, comprehensive evaluation results of every floor as well as the final comprehensive evaluation results can be acquired.

Fuzzy comprehensive evaluation model needs consider economic benefits, social benefits and technical benefits. Evaluation indexes of comprehensive evaluation are shown in Table 2. In view of benefit evaluation index of Interaction between micro grid and distribution network, firstly, index weight was determined by analytic hierarchy process, secondly, build up decision matrix by fuzzy comprehensive evaluation to carry out the evaluation, at last, comprehensive benefit of different interaction models were analyzed according to fuzzy comprehensive evaluation to determine the comprehensive benefit based on interaction of micro grid and distribution network, adopting maximum membership degree law.

Table 2. Comprehensive Benefit Evaluation Index System of the Grid

\begin{tabular}{cc}
\hline first grade indexes & second grade indexes \\
\hline & peak load shifting A1 \\
reduce fuel cost A2 \\
reduce investment costA3 \\
selling benefit of the grid A4 \\
delay electric grid invests A5 \\
reduce spinning reserve A6 \\
environmental benefit B1 \\
Social benefits B & promote energy conversion efficiency B2 \\
& reduce electric cost expenditure of users B3 \\
Technical benefits C & power supply reliability C1 \\
& loss reduction C2 \\
& power quality C3
\end{tabular}

\section{Example Analysis of Fuzzy Comprehensive Evaluation}

Take a distribution network connected with micro grid for example, system wiring is shown in picture $2^{[21]}$. 


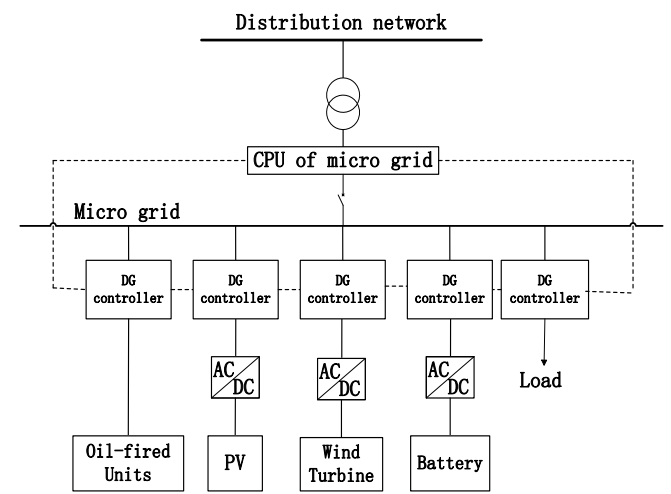

Picture 2. System Diagram of the Grid

\subsection{Interaction Model based on Economic Goal}

(1) Judgment matrix of the total evaluation index is as below:

$$
\begin{aligned}
& \begin{array}{lll}
A & B & C
\end{array} \\
& \begin{array}{l}
A \\
B
\end{array} \quad\left(\begin{array}{ccc}
1 & 2 & 5 \\
1 / 2 & 1 & 2 \\
1 / 5 & 1 / 2 & 1
\end{array}\right)
\end{aligned}
$$

The methods of calculating weight vector and characteristic root are "sum-product method", "square root method" and "root method". Concerning that "sum-product method" is easy to calculate, so it is adopted in this paper.

$$
A^{\prime}=\left(\begin{array}{ccc}
0.5882 & 0.5714 & 0.625 \\
0.2941 & 0.2857 & 0.25 \\
0.1176 & 0.1429 & 10.125
\end{array}\right)
$$

Weight vector $\mathrm{W}=(0.5949,0.2766,0.1285)^{\mathrm{T}}$

\begin{tabular}{|c|c|c|c|c|c|c|}
\hline & $A 1$ & $A 2$ & $A 3$ & $A 4$ & $A 5$ & $A 6$ \\
\hline$A 1$ & 1 & 2 & 3 & 3 & 4 & 5 \\
\hline$A 2$ & $1 / 2$ & 1 & 2 & 2 & 3 & 3 \\
\hline$A 3$ & $1 / 3$ & $1 / 2$ & 1 & 1 & 1 & 2 \\
\hline$A 4$ & $1 / 3$ & $1 / 2$ & 1 & 1 & $1 / 2$ & $1 / 2$ \\
\hline$A 5$ & $1 / 4$ & $1 / 3$ & 1 & 2 & 1 & 1 \\
\hline A6 & $1 / 5$ & $1 / 3$ & $1 / 2$ & 2 & 1 & 1 \\
\hline
\end{tabular}

The results are reliable with consistency Check.

(2) Judgment matrix is as below:

Weight vector can be calculated: $\mathrm{W} 2=(\mathrm{A} 1, \mathrm{~A} 2, \mathrm{~A} 3, \mathrm{~A} 4, \mathrm{~A} 5, \mathrm{~A} 6) \mathrm{T}=(0.3589,0.2182,0.113$, $0.1148,0.1038,0.0912) \mathrm{T}$ 。

With consistency, maximum characteristic root $\lambda_{\max }=6.3568$.

(3) Judgment matrix of social benefits is as below:

$$
\begin{array}{lll}
B 1 & B 2 & B 3
\end{array}
$$

$B 1$
$B 2$
$B 3$$\quad\left(\begin{array}{ccc}1 & 3 & 5 \\ 1 / 3 & 1 & 2 \\ 1 / 5 & 1 / 2 & 1\end{array}\right)$

Weight vector can be calculated: $\mathrm{W} 3=(\mathrm{B} 1, \mathrm{~B} 2, \mathrm{~B} 3)^{\mathrm{T}}=(0.648,0.2299,0.1222,)^{\mathrm{T}}$; With consistency, maximum characteristic root $\lambda_{\max }=3.0037$.

(4) Judgment matrix of technical benefits is as below: 


$$
\begin{aligned}
& \begin{array}{lll}
C 1 & C 2 & C 3
\end{array} \\
& \begin{array}{l}
C 1 \\
C 2 \\
C 3
\end{array} \quad\left(\begin{array}{ccc}
1 & 2 & 4 \\
1 / 2 & 1 & 5 \\
1 / 4 & 1 / 5 & 1
\end{array}\right)
\end{aligned}
$$

Weight vector can be calculated: $\mathrm{W} 3=(\mathrm{C} 1, \mathrm{C} 2, \mathrm{C} 3)^{\mathrm{T}}=(0.532,0.3661,0.1018)^{\mathrm{T}}$,; With consistency, maximum characteristic root $\lambda_{\max }=3.0947$.

(5) Combination weight is designed as below:

Weight of first grade indexes can be calculated: $\mathrm{W}=(0.5949,0.2766,0.1285) \mathrm{T}$ 。

Combination weight of first grade indexes can be calculated:

$$
\mathrm{W}^{\prime}=\left\{\begin{array}{rrr}
0.3589 & 0 & 0 \\
0.2182 & 0 & 0 \\
0.1130 & 0 & 0 \\
0.1148 & 0 & 0 \\
0.1038 & 0 & 0 \\
0.0912 & 0 & 0 \\
0 & 0.6480 & 0 \\
0 & 0.2299 & 0 \\
0 & 0.1222 & 0 \\
0 & 0 & 0.5321 \\
0 & 0 & 0.3661 \\
0 & 0 & 0.1018
\end{array}\right\} * W=\left\{\begin{array}{l}
0.2135 \\
0.1298 \\
0.0672 \\
0.0683 \\
0.0618 \\
0.0542 \\
0.1792 \\
0.0636 \\
0.0338 \\
0.0684 \\
0.0470 \\
0.0131
\end{array}\right\}
$$

From the arithmetic expression above, specific evaluation results of comprehensive benefit based on interaction of micro grid and distribution network are shown in table 3 .

Table 3. Evaluation Index System of Comprehensive Benefit

\begin{tabular}{cccc}
\hline first grade indexes and weights & second grade indexes and weights \\
\hline & & peak load shifting A1 & 0.214 \\
Economic benefits A & 0.5949 & reduce fuel cost A2 & 0.130 \\
& & reduce investment costA3 & 0.067 \\
& & selling benefit of the grid A4 & 0.068 \\
Social benefits B & \multirow{2}{*}{0.2766} & delay electric grid invests A5 & 0.062 \\
& & reduce spinning reserve A6 & 0.054 \\
& & promote energy conversion efficiency B2 & 0.063 \\
Technical benefits C & 0.1258 & reduce electric cost expenditure of users B3 & 0.034 \\
& & power supply reliability C1 & 0.065 \\
& & loss reduction C2 & 0.047 \\
& & power quality C3 & 0.013 \\
\hline
\end{tabular}

(6) The calculation of fuzzy comprehensive evaluation

Single factor evaluation results of economic benefits are shown in table 4:

Table 4. Single Factor Evaluation Results of Economic Benefits

\begin{tabular}{llllll}
\hline factor set & good & well & $\begin{array}{c}\text { comment set } \\
\text { medium }\end{array}$ & poor & bad \\
\hline $\begin{array}{c}\text { peak load shifting } \\
\text { A1 }\end{array}$ & 0.65 & 0.22 & 0.129 & 0 & 0 \\
reduce fuel cost A2 & 0.60 & 0.192 & 0.123 & 0.060 & 0.02 \\
\hline
\end{tabular}




\begin{tabular}{cccccc}
\hline $\begin{array}{c}\text { reduce investment } \\
\text { cost A3 }\end{array}$ & 0.46 & 0.235 & 0.141 & 0.116 & 0.05 \\
$\begin{array}{c}\text { selling benefit of } \\
\text { the grid A4 }\end{array}$ & 0.45 & 0.302 & 0.180 & 0.065 & 0 \\
$\begin{array}{c}\text { delay electric grid } \\
\text { invests A5 }\end{array}$ & 0.80 & 0.144 & 0.015 & 0.043 & 0 \\
$\begin{array}{c}\text { reduce spinning } \\
\text { reserve A6 }\end{array}$ & 0.77 & 0.116 & 0.056 & 0.038 & 0.02 \\
\hline
\end{tabular}

Single factor evaluation matrix of economic benefits is as below:

$$
R 1=\left[\begin{array}{lllll}
0.651 & 0.220 & 0.129 & 0.000 & 0.000 \\
0.604 & 0.192 & 0.123 & 0.060 & 0.021 \\
0.458 & 0.235 & 0.141 & 0.116 & 0.050 \\
0.453 & 0.302 & 0.180 & 0.065 & 0.000 \\
0.798 & 0.144 & 0.015 & 0.043 & 0.000 \\
0.769 & 0.116 & 0.056 & 0.038 & 0.021
\end{array}\right]
$$

Evaluation results vector of economic benefits:

$\mathrm{B} 1=(0.6222,0.1936,0.1164,0.0416,0.0121)^{\mathrm{T}}$

Single factor evaluation results of social benefits are shown in Table 5:

Table 5. Single Factor Evaluation Results of Social Benefits

\begin{tabular}{cccccl}
\hline factor set & \multicolumn{5}{c}{ comment set } \\
& good & well & medium & poor & bad \\
\hline Environmental benefit B1 & 0.521 & 0.312 & 0.089 & 0.078 & 0 \\
Promote energy conversion efficiency B2 & 0.774 & 0.125 & 0.074 & 0.027 & 0 \\
Reduce electric cost expenditure of users B3 & 0.499 & 0.345 & 0.130 & 0.026 & 0 \\
\hline
\end{tabular}

Single factor evaluation matrix of social benefits is as below:

$$
R 2=\left[\begin{array}{ccccc}
0.521 & 0.312 & 0.089 & 0.078 & 0.000 \\
0.774 & 0.125 & 0.074 & 0.027 & 0.000 \\
0.499 & 0.345 & 0.130 & 0.026 & 0.000
\end{array}\right]
$$

Evaluation results vector of social benefits:

$\mathrm{B} 2=(0.5765,0.2731,0.0906,0.0600,0.0000)^{\mathrm{T}}$

Single factor evaluation results of technical benefits are shown in table 6:

\begin{tabular}{|c|c|c|c|c|c|}
\hline \multirow{2}{*}{ factor set } & \multicolumn{5}{|c|}{ comment set } \\
\hline & good & well & medium & poor & bad \\
\hline power supply reliability $\mathrm{C} 1$ & 0.598 & 0.302 & 0.090 & 0.006 & 0.004 \\
\hline loss reduction $\mathrm{C} 2$ & 0.769 & 0.162 & 0.014 & 0.053 & 0 \\
\hline power quality $\mathrm{C} 3$ & 0.479 & 0.316 & 0.096 & 0.089 & 0.02 \\
\hline
\end{tabular}

Table 6. Single Factor Evaluation Results of Technical Benefits

Single factor evaluation matrix of technical benefits is as below:

$$
R 3=\left[\begin{array}{ccccc}
0.598 & 0.302 & 0.090 & 0.006 & 0.004 \\
0.769 & 0.162 & 0.014 & 0.053 & 0.000 \\
0.479 & 0.316 & 0.096 & 0.089 & 0.020
\end{array}\right]
$$


Evaluation results vector of technical benefits:

B3 $=(0.6485,0.2522,0.0628,0.0316,0.0042)^{\mathrm{T}}$

Evaluation results of comprehensive benefit based on interaction of micro grid and distribution network are shown in table 7:

Table 7. Single Factor Evaluation Results of Fuzzy Evaluation

\begin{tabular}{cccccc}
\hline factor set & good & well & medium & poor & bad \\
\hline Ecnomic benefits & 0.622 & 0.194 & 0.116 & 0.041 & 0.012 \\
Social benefits & 0.577 & 0.273 & 0.091 & 0.060 & 0.000 \\
\hline Technical benefits & 0.649 & 0.252 & 0.063 & 0.032 & 0.005
\end{tabular}

The total evaluation matrix $\mathrm{R}$ is made up of $\mathrm{B} 1, \mathrm{~B} 2, \mathrm{~B} 3$ as rows:

$$
R=\left[\begin{array}{ccccc}
0.6222 & 0.1936 & 0.1164 & 0.0416 & 0.0121 \\
0.5765 & 0.2731 & 0.0906 & 0.0600 & 0.0000 \\
0.6485 & 0.2522 & 0.0628 & 0.0316 & 0.0042
\end{array}\right]
$$

Comprehensive benefit vector based on interaction of micro grid and distribution network: $\mathrm{B}=(0.6129,0.2231,0.1024,0.0454,0.0077)^{\mathrm{T}}$

Corresponding columnar section is shown in picture 3 :

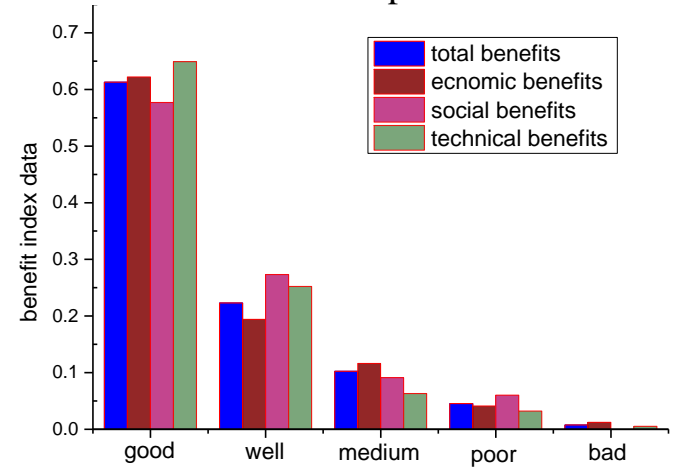

\section{Picture 3. Columnar Section of Comprehensive Benefit}

In the final comprehensive evaluation results, membership degree belongs to "good" is 0.6129 , the sum of membership degrees belongs to "good" and "well" is 0.836 , according to maximum membership degree law, the answer is satisfactory, so, interaction of micro grid and distribution network has comprehensive significant benefits.

\subsection{Interaction Model based on Social Goal}

Judgment matrix of the total evaluation index is as below:

$$
\begin{gathered}
A \\
A \\
B \\
C
\end{gathered} \quad\left(\begin{array}{ccc}
1 & 1 / 5 & 1 / 2 \\
5 & 1 & 2 \\
2 & 1 / 2 & 1
\end{array}\right)
$$

Weight vector $\mathrm{W}=(0.1285,0.5949,0.2766)^{\mathrm{T}}$

The results are reliable with consistency Check.

Weight of first grade indexes can be calculated: $\mathrm{W}=(0.1285,0.5949,0.2766)^{\mathrm{T}}$.

Combination weight of first grade indexes can be calculated: 


$$
\mathrm{W}^{\prime}=\left\{\begin{array}{rrr}
0.3589 & 0 & 0 \\
0.2182 & 0 & 0 \\
0.1130 & 0 & 0 \\
0.1148 & 0 & 0 \\
0.1038 & 0 & 0 \\
0.0912 & 0 & 0 \\
0 & 0.6480 & 0 \\
0 & 0.2299 & 0 \\
0 & 0.1222 & 0 \\
0 & 0 & 0.5321 \\
0 & 0 & 0.3661 \\
0 & 0 & 0.1018
\end{array}\right\} * W=\left\{\begin{array}{l}
0.0461 \\
0.0280 \\
0.0145 \\
0.0148 \\
0.0133 \\
0.0117 \\
0.3855 \\
0.1368 \\
0.0727 \\
0.1472 \\
0.1013 \\
0.0282
\end{array}\right\}
$$

Specific evaluation results of comprehensive benefit based on interaction of micro grid and distribution network are shown in Table 8.

Table 8. Evaluation Index System of Comprehensive Benefit

\begin{tabular}{llll}
\hline first grade indexes and weights & second grade indexes and weights & \\
Economic benefits A & 0.1285 & peak load shifting A1 & 0.0461 \\
& & reduce fuel cost A2 & 0.0280 \\
& & reduce investment costA3 & 0.0145 \\
& selling benefit of the grid A4 & 0.0148 \\
& & delay electric grid invests A5 & 0.0133 \\
Social benefits B & \multirow{2}{*}{0.5949} & reduce spinning reserve A6 & 0.0117 \\
& & environmental benefit B1 & 0.3855 \\
Technical benefits C & \multirow{2}{*}{0.2766} & promote energy conversion efficiency B2 & 0.1368 \\
& & power supply reliability C1 & 0.0727 \\
& & poss reduction C2 & 0.1472 \\
& & & 0.1013 \\
& &
\end{tabular}

Comprehensive benefit vector based on interaction of micro grid and distribution network: $\mathrm{B}=(0.6011,0.2571,0.0862,0.0498,0.0027)^{\mathrm{T}}$

Corresponding columnar section is shown in picture 4 :

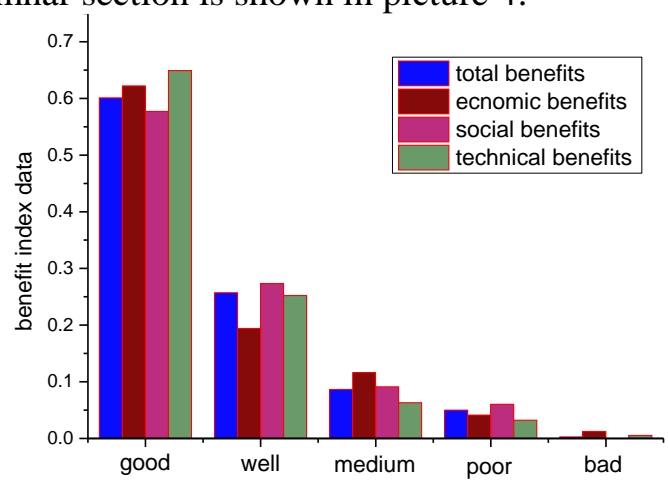

Picture 4. Columnar Section of Comprehensive Benefit

In the final comprehensive evaluation results, membership degree belongs to "good" is 0.6011 , the sum of membership degrees belongs to "good" and "well" is 0.8582 , according to maximum membership degree law, the answer is satisfactory, so, interaction of micro grid and distribution network has comprehensive significant benefits. 


\subsection{Interaction Model based on Technical Goal}

Judgment matrix of the total evaluation index is as below:

$$
\begin{aligned}
& \begin{array}{lll}
A & B & C
\end{array} \\
& \begin{array}{l}
A \\
B \\
C
\end{array} \quad\left(\begin{array}{ccc}
1 & 1 / 3 & 1 / 5 \\
3 & 1 & 1 / 2 \\
5 & 2 & 1
\end{array}\right)
\end{aligned}
$$

Weight vector $\mathrm{W}=(0.1096,0.3091,0.5812)^{\mathrm{T}}$

The results are reliable with consistency Check.

Weight of first grade indexes can be calculated: $\mathrm{W}=(0.1096,0.3091,0.5812) \mathrm{T}$.

Combination weight of first grade indexes can be calculated:

$$
\mathrm{W}^{\prime}=\left\{\begin{array}{rrr}
0.3589 & 0 & 0 \\
0.2182 & 0 & 0 \\
0.1130 & 0 & 0 \\
0.1148 & 0 & 0 \\
0.1038 & 0 & 0 \\
0.0912 & 0 & 0 \\
0 & 0.6480 & 0 \\
0 & 0.2299 & 0 \\
0 & 0.1222 & 0 \\
0 & 0 & 0.5321 \\
0 & 0 & 0.3661 \\
0 & 0 & 0.1018
\end{array}\right\} * W=\left\{\begin{array}{l}
0.0393 \\
0.0239 \\
0.0124 \\
0.0126 \\
0.0114 \\
0.0010 \\
0.2003 \\
0.0711 \\
0.0378 \\
0.3093 \\
0.2128 \\
0.0592
\end{array}\right\}
$$

Specific evaluation results of comprehensive benefit based on interaction of micro grid and distribution network are shown in table 9.

Table 9. Evaluation Index System of Comprehensive Benefit

\begin{tabular}{cccc}
\hline first grade indexes and weights & second grade indexes and weights \\
\hline & & peak load shifting A1 & 0.0393 \\
Economic benefits A & 0.1096 & reduce fuel cost A2 & 0.0239 \\
& & reduce investment costA3 & 0.0124 \\
& & selling benefit of the grid A4 & 0.0126 \\
& & delay electric grid invests A5 & 0.0114 \\
Social benefits B & \multirow{2}{*}{0.3091} & reduce spinning reserve A6 & 0.0010 \\
& & environmental benefit B1 & 0.2003 \\
Technical benefits C & \multirow{2}{*}{0.5812} & reduce electric cost expenditure of users B3 & 0.0378 \\
& & power supply reliability C1 & 0.3093 \\
& & loss reduction C2 & 0.2128 \\
& & power quality C3 & 0.0592 \\
\hline
\end{tabular}

Comprehensive benefit vector based on interaction of micro grid and distribution network: $\mathrm{B}=(0.6233,0.2522,0.0773,0.0415,0.0038)^{\mathrm{T}}$

Corresponding columnar section is shown in Picture 5: 


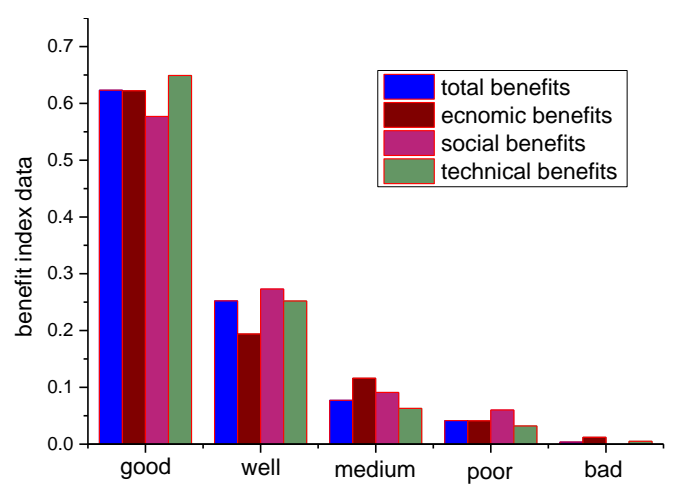

\section{Picture 5. Columnar Section of Comprehensive Benefit}

In the final comprehensive evaluation results, membership degree belongs to "good" is 0.6233 , the sum of membership degrees belongs to "good" and "well" is 0.8755 , according to maximum membership degree law, the answer is satisfactory, so, interaction of micro grid and distribution network has comprehensive significant benefits.

\section{Solution}

Interaction of micro grid and distribution network is the important link for china to build up forceful smart grid. In this paper, three types of interaction between micro grid and distribution network were determined, comprehensive benefits evaluation of which was carried. Firstly, index system of comprehensive benefit was determined, then index weight was determined by analytic hierarchy process, at last, comprehensive benefit of different interaction models were analyzed according to fuzzy comprehensive evaluation.

Example analysis shows that construction investment cost of Interaction of micro grid and distribution network is a little high, but in views of the whole society, think about improving reliability, energy saving, environmental protection and delaying electric grid invests, interaction of micro grid and distribution network produces much benefits. As for interaction model based on economic goal, interaction model based on social goal and interaction model based on technical goal, in the final comprehensive evaluation results, membership degree belongs to "good" is the biggest, what's more, membership degree belongs to "good" is bigger than 0.5 for all three interaction models, according to maximum membership degree law, the grid has significant comprehensive benefits. In addition, the analysis shows that membership degree belongs to "good" of interaction model based on technical goal is bigger than other two interaction models, which has the most significant comprehensive benefits.

\section{References}

[1] XI O Jun, C Yanyvdffvcan, W Jianmin, "A Hierarchical Performance Assessment Method on the Distribution Network Planning". vol. 32, no. 15, (2008), pp. 36-40.

[2] Z-h Tan, L Ge, Q-p Sun, "Simplified model of distribution network based on minimum area and its application", Electricity.

[3] T Ya-fang, W Jian-min, C Hao-zhong, (2007),(S2), pp. 127-130.

[4] W. Qi, L. Xintao, L. Changyuan,e $t$ al, "Research on the combined control method for parallel inverters control of micro-grid", Strategic Technology (IFOST), 2012 7th International Forum on, (2012), pp. 1-5.

[5] C. Yuan, "Study on the Structure and Function of the Distribution Network System Containing Micro-Grid[J]", vol. , no. 1, (2008), pp. 34-39.

[6] Z Zhang, Y Li, W Chen, "The research on micro-grid mode conversion", Electricity Distribution (CICED), 2012 China International Conference on, (2012), pp. 1-7.

[7] X Dong, L Jiao, L Tan, "Comprehensive economic benefits evaluation of the micro grid", Technology Wind, (2013), no. 19, pp. 217-218.

[8] X Xiang, Y Yue, L Zhenjie Hohai University, "Reliability Analysis of a Novel Distribution Network with Microgrid[J]". vol. 35, no. 9, (2011), pp. 67-72. 
[9] Z Ming, Z Xiaohui, J Xiaoxi, "Benefit analysis and evaluation model application of smart micro grid technologies", no. 05, (2013), pp. 173-175.

[10] A. Xin, X. Jia-jia, "Study on the microgrid and distribution network co-operation model based on interactive scheduling", vol. 41, no. 1, (2013), pp. 143-149.

[11] T. T Erbato, T Hartkopf. "Smarter Micro Grid for energy solution to rural Ethiopia[C]", Innovative Smart Grid Technologies (ISGT), 2012 IEEE PES, (2012), pp. 1-7.

[12] Q Ye, T Ma, Y Jun Gu, "Research on dispatch scheduling model of micro-grid with distributed energy[C]". Electricity Distribution (CICED), 2012 China International Conference on, (2012), pp. 1-5.

[13] H Peng, A Xin, X Hong, "Overview of the Economic Operation of Microgrid", 2012, vol. 29, no. 4, pp. 1-6.

[14] L Shuai-qi, M Xiao-chun, Z Xue, "Operation and control techniques of Micro-Grids with energy storage[J]”, vol. 26, no. 4, (2011), pp. 74-79.

[15] P. Kessel, H Glavitsch, "Estimating the voltage stability of a power system[J]". Power Delivery, IEEE Transactions on, vol. 1, no. 3, (1986), pp. 346-354.

[16] F. Katiraei, Iravani M R, Lehn P W, "Micro-grid autonomous operation during and subsequent to islanding process[J]", Power Delivery, IEEE Transactions on, vol. 20, no. 1, (2005), pp. 248-257.

[17] T L Saaty, "Decision making with the analytic hierarchy process[J]", International journal of services sciences, vol. 1, no. 1, (2008), pp. 83-98.

[18] Z Jian, C Yan-fen, Y Qi-zhi,"Research on Service Platform of Three Gorges Lockage Maritime Security[C]", Distributed Computing and Applications to Business, Engineering \& Science (DCABES), 2012 11th International Symposium on, (2012), pp. 362-365.

[19] S. Ji , X Li, Y Ma, "Optimal tolerance allocation based on fuzzy comprehensive evaluation and genetic algorithm”, The International Journal of Advanced Manufacturing Technology, (2000), vol. 16, no. 7, pp. 461-468.

[20] W Jing-min, S Yan fu, "The Application of Multi-Level Fuzzy Comprehensive Evaluation Method in Technical and Economic Evaluation of Distribution Network[C]", Management and Service Science (MASS), 2010 International Conference on, (2010), pp. 1-4.

[21] Y Qi, M Shiying, T Xiaojun, "Evaluation Index System Construction and Application of Microgrid Planning", vol. 3, no. 9, (2012), pp. 13-17.

\section{Authors}

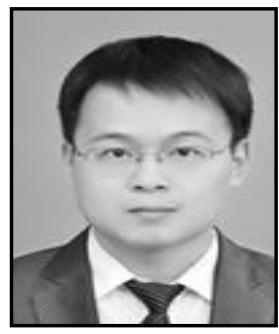

Weiping Zhu:(1983 - $)$, male, doctor, majored in the research of post - fault analysis in power distribution systems; email: zwp_xjtu@163.com

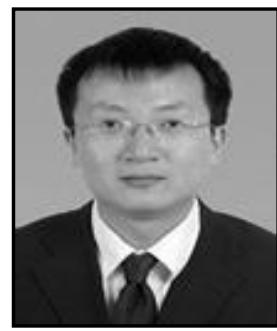

Xiaodong Yuan: $(1979-)$, male, senior engineer, majored in the research of Power Performance and Power Quality; email: lnnayUyna@hotmail.eom

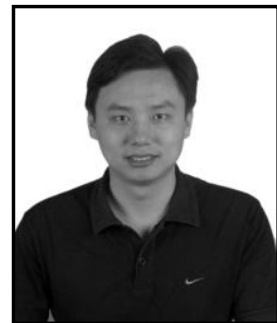

Yunpeng Li: (1978 -), male, senior engineer , majored in the research of information management and technical work of power System Automation ; email: leeyunpeng@sina.com 